\title{
Pervasive Recommender System for Smart Home Environment
}

\author{
Naouar Belghini \\ TIC Team, LSI Lab, \\ ESTEM Research Center \\ Casablanca, Morocco
}

\author{
Nesrine Gouttaya \\ TIC Team, LSI lab, \\ ESTEM Research Center \\ Casablanca, Morocco
}

\author{
Wafaâ Bouab \\ Bennani \\ TIC Team, LSI Lab, \\ ESTEM Research Center
}

\author{
Adil Sayouti \\ Team Architecture of \\ Systems \\ LISER Laboratory \\ ENSEM, Hassan II \\ University
}

\begin{abstract}
Buildings today become complex in terms of structures and advanced technologies. Intelligent buildings are autonomic environment that provide useful services to make occupants lives' more comfortable (e.g. physical security, automatic lighting, thermal comfort, etc...). Smart buildings use IT systems to connect a variety of independent sub-systems, so that these systems can share information to improve total building performance.

In this paper, we propose an approach that offers the most relevant services to inhabitants according to the contextual information provided by the physical sensors. The proposed approach considers five parameters to represent the contextual information: the user activity (sleeping, walking, sitting down, ...), time(morning, afternoon, night, ...) localization ( kitchen, living room,..), temperature(warm, cold, hot, ...), special event (ween end, holidays,...) and activate the most adequate service to be applied in a given context of use.
\end{abstract}

\section{Keywords}

Smart home, pervasive computing, recommender systems, human activity recognition, neural network

\section{INTRODUCTION}

Advances in nanotechnologies and computer sciences over the last decade have made electronic devices smart, cheaper and smaller. Also, revolutions made in communication technology, has strongly contributed to the improvement of innovative networked systems. It is in this context that pervasive (called also context-aware) systems have been emerged and developed. These systems include a collection of various devices embedded in the physical environment that allow system's self-management to achieve autonomic and personalized functionalities. They are emerging solutions that provide a way to adapt the behavior of applications in order to satisfy user expectations. Context-aware systems require the capacity to perceive the current situation of the user, predict his needs and act spontaneously by offering the most appropriate services taking into account his contextual situation.

Smart building system (or building automation) has received considerable attention from computer industry in the past decade. It can be defined as a "supersystem" of interconnected building subsystems that refers to any automatic control system used to controls buildings lighting, ventilation, air conditioning, etc....

In fact, smart home research area presents an important context aware research area. Its aim is to improve occupant comfort by offering automatic and personalized assistance to inhabitant.

Many applications and projects have been developed in this field. Authors in [1] have proposed a Smart Home Inhabitant Prediction algorithm to expect the next action of a user. The algorithm identifies matching sequences from the collected history and uses them to predict the next command. Gopalratnam and Cook present in [2] a system to predict arbitrary inhabitant-home interactions; the proposed system predicts which device the user will interact with next. Katharina Rasch in [3] proposed two smart assistants for smart homes. The first assistant was a recommender system that suggests useful services for the user. The suggested services are tailored to the user's current situation, behaviors and preferences. The second assistant addressed the installation procedures. It reduces the need for manually describing device functionalities. As an alternative, users can simply plug in a new device and let the installation assistant run automatically. In [4] the authors propose an approach to present spontaneously and continuously the post relevant services the user in smart environment in response to any significant change of his context. They use the cased based reasoning method to stock user interactions with his smart environments and use this history by artificial neural network to predict him relevant services in future context of use even in new context situation.

The purpose of this paper is to propose an approach that enables intelligent home to become context-aware.

The paper is organized as follows: Section 2 presents the state of the art about the evolution of Pervasive Recommender Systems. Section 3 introduces the definition of the contextual information. In section 4 , we give a general overview about human activity recognition in intelligent home environment. And, in section 5, we present the principle of the proposed solution.

\section{THE EVOLUTION OF PERVASIVE RECOMMENDER SYSTEMS}

\subsection{Recommender Systems}

Recommender systems can be considered as a kind of Information Retrieval system providing personalized recommendations to users. They intend to facilitate research and access to information by offering suitable services. The principle of recommender systems is based on the process of filtering and adapting incoming information from users [5][6].

However, traditional recommender systems, such as those based on content-based and collaborative filtering, tend to use 
fairly simple user models. For example, user-based collaborative filtering generally models the user as a vector of item ratings. As additional observations are made about users' preferences, the user models are extended, and the full collection of user preferences is used to generate recommendations or make predictions. This approach, therefore, ignores the notion of "situated actions", the fact that users interact with the system within a particular "context" and that preferences for items within one context may be different from those in another context.

\subsection{Pervasive Systems}

Regarding the pervasive Systems [7], they extend the Information System paradigm by introducing a set of novel characteristics and making services available anytime, anywhere and from any device. Contrary to traditional information systems, Pervasive systems have to support a multitude of heterogeneous device and offering users appropriate services considering their goals and the context in which such goals appear, as well as the capability of anticipating upcoming goals in this context [8].

The main challenge of pervasive systems is to capture and model user's intention to assist him in his daily life by providing proactively appropriate services at the right time : without "direct" intervention from him. Indeed, the pervasive environment must be no longer just to "react" to user commands but rather "acting".

In order to overcome limitations of the traditional recommender systems in pervasive environment. The pervasive recommender systems have been proposed.

\subsection{Pervasive Recommender systems}

Pervasive Recommender Systems (PRS) combine the characteristics of pervasive systems and traditional recommender systems to provide proactively personalized user recommendations in pervasive environments.

In our vision, a Pervasive Recommender System should have the characteristics that we define by:

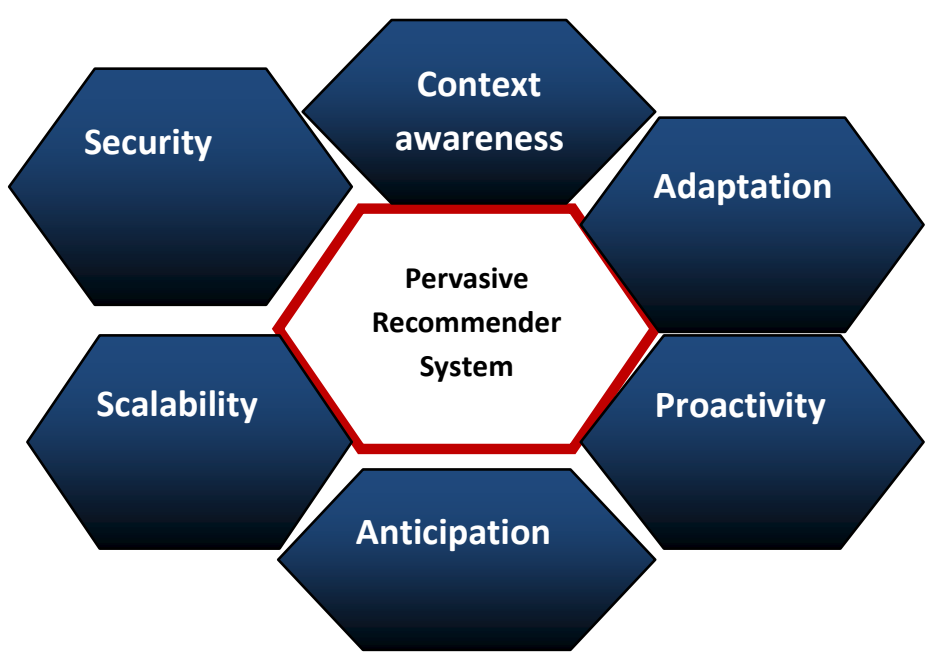

Fig 1: Characteristics of Pervasive Recommender Systems

\section{- Context Awareness}

A pervasive recommender system must provide continuous perception of all its sensors to collect contextual information(temperature, light, motion, etc) in real time, interpreting these parameters to extract high-context information level, such as the identification of the user's activity (meeting, running errands, etc.) and finally use this information to adapt his behavior to different changes that may occur in the future on this context.

\section{- Adaptation}

The process with which a pervasive recommender system adapts its behavior to the needs and preferences of its users is called adaptation. Adaptation is based on user related and context related information.

\section{- Proactivity}

In addition to the ability of context awareness and context aware adaptation, pervasive recommender systems need to be proactive. In other words, they must be able to provide, spontaneously, personalized services to the user in order to minimize his/her explicit interaction with the intelligent environment.

\section{- Anticipation}

Pervasive Recommender systems must be able to anticipate future contexts of use and future user needs and preferences in these new contextual situations in order to serve the user continuously (in an uninterrupted manner).

\section{- Scalability}

Pervasive Recommender systems must be also able to evolve over time in order to support any changes that may arise in user's context or/an in user's needs and preferences in terms of intelligent services.

\section{- Security}

Pervasive recommender system must be able to protect the personal data of users against destruction, theft or unauthorized access.

\section{CONTEXT ANALYSIS}

In computing world, many definitions have been proposed in the literature to define context and context-awareness. The Most adopted definitions are:

"Any information that can be used to characterize the situation of entities (whether a person, place or object) that are considered relevant to the interaction between a user and an application, including the user and the application themselves." [9].

"Context is not simply the state of a predefined environment with a fixed set of interaction resources. It is part of a process of interacting with an ever-changing environment composed of reconfigurable, migratory, distributed, and multiscale resources [10]."

Pervasive systems typically consider many parameters to extract contextual information:

Table 1: Example of Some Possible Contextual Parameters

\begin{tabular}{|c|c|}
\hline Category & interpretation \\
\hline $\begin{array}{c}\text { User } \\
\text { context }\end{array}$ & $\begin{array}{l}\text { User's profile (who?): identifications, } \\
\text { interests, etc }\end{array}$ \\
\hline $\begin{array}{c}\text { Physical } \\
\text { context }\end{array}$ & $\begin{array}{l}\text { Physical Environment: temperature, color, } \\
\text { time of day, etc }\end{array}$ \\
\hline $\begin{array}{l}\text { Network } \\
\text { context }\end{array}$ & $\begin{array}{l}\text { Network environment: connectivity, protocol, } \\
\text { etc }\end{array}$ \\
\hline
\end{tabular}




\begin{tabular}{|c|c|}
\hline $\begin{array}{c}\text { Activity } \\
\text { context }\end{array}$ & What occurs, at what time: enter, go out, etc \\
\hline $\begin{array}{c}\text { Service } \\
\text { context }\end{array}$ & $\begin{array}{c}\text { Information on functions which system can } \\
\text { provide: turn TV on a preferred channel, turn } \\
\text { light off, etc... }\end{array}$ \\
\hline
\end{tabular}

So, in order to enable an efficient context-aware adaptation, context information must be well captured, collected and presented.

Many studies have been done in this context. Manzoor et al. [11] quantify the Quality of Context parameters to be used in pervasive environments. They also present a mechanism to tailor the Quality of Context (QoC) parameters according to a specific need of an application and then evaluate these parameters.

To simplify the programming issue of context-aware systems it is necessary to adopt a modeling method to represent the context information. This is very important to capture user's profiles and except their needs.

The most relevant context modeling approaches are classified by the structure of data used to exchange contextual information in the pervasive system. They are based on one of the following methods:

\section{- Key-Value Models}

The model of key-value pairs is the most simple data structure for modeling contextual information. A context is described by a set of two dimensional vectors. Each vector consists of a numerical value describing the situations and a number indicating the certainty that the user is currently in this situation.

\section{- Markup Scheme Models}

It is a hierarchical data structure consisting of markup tags (ex. XML). An example of this approach is the Comprehensive Structured Context Profiles by Held et al. [12].

\section{- Graphical Models}

A well known modeling instrument is the Unified Modeling Language (UML) which has a strong graphical component (UML diagrams). Due to its generic structure, UML is also appropriate to model the context.

\section{- Logic Based Models}

A logic defines the conditions on which a concluding expression (or fact) may be extracted from a set of expressions or facts. In a logic based context model, the context is defined as facts, expressions and rules.

\section{- Ontology Based Models}

Ontologies are widely accepted as instrument for the modeling of context information in pervasive computing applications.

In the context of computer and information sciences, an ontology defines a set of representational primitives used to model a domain of knowledge. The representational primitives are typically classes, properties, and relations among class members. It is a promising instrument to define explicit formal specifications of the terms in a domain and the relations among them.

A detailed survey of these models is presented in [13].

\section{HUMAN ACTIVITY RECOGNITION}

Activity recognition systems refer to systems based on sensors (like cameras, wearables, wireless sensor networks, etc...) that allow obtaining and interpreting information to recognize which activities are performed.

Especially in the context of home automation, human activity identification provides important information in the process of analyzing the contextual information of inhabitants and then proposing the suitable actions to be proposed or executed.
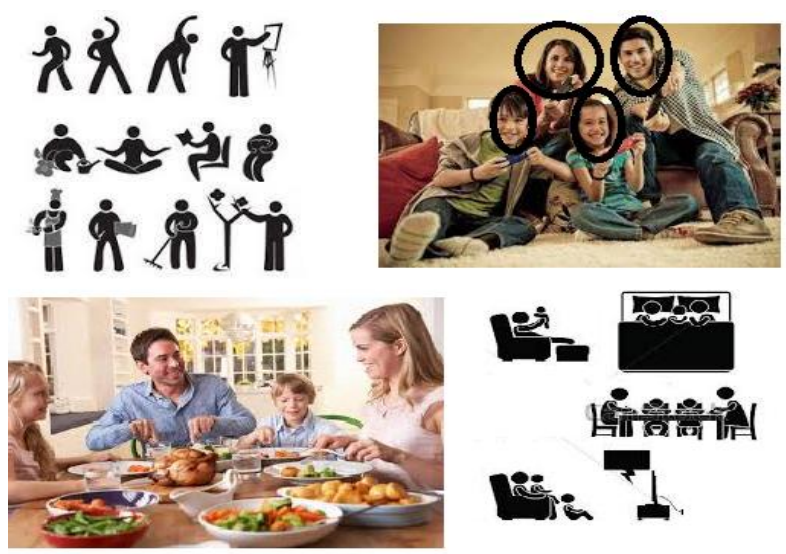

Fig 2: Example of daily human activities

A detailed state-of-the-art research is presented in [14], the research discusses the complexity of pervasive computing in terms of highly sensorised environments and contextual applications customized to a variety of factors. Authors provide a comprehensive analysis of the nature and characteristics of situations, discuss the complexities of situation identification, and review the techniques that are most popularly used in modelling and inferring situations from sensor data.

Several other studies have been done to recognize and identify human activities in smart home environment. And most notable results were obtained using probabilistic models based on hidden Markov model (HMM). In [15], authors use semi hidden Markov Models to recognize activities from sensors. The some authors in [16] addresses the problem of how to use annotated training data from one environment to label training data from another environment. They introduce the use of a transfer learning method for activity recognition, which transfer knowledge about activity recognition and allows the use of labelled and unlabeled data to learn house-specific behavior. However, details of activities may vary significantly from person to person and from home to home in which case this technique may not perform well.

In another study[17], authors present an Activity recognition system for single person smart homes using Active Learning in the presence of Overlapped activities. The proposed system applies data mining techniques to cluster in-home sensor firings so that each cluster represents instances of the same activity. Once the clusters are associated to their corresponding activities, the system can recognize future activities.

Recently, Dragan et all [18] presents a method for image based human activity recognition, in a smart environment. Authors use background subtraction and skeletisation as image processing techniques, combined with Artificial Neural 
Networks for human posture classification and Hidden Markov Models for activity interpretation.

\section{THE PROPOSED APPROACH}

\subsection{Principle}

In the context of the daily living, we remark that different activities are performed in different rooms, each of them triggers a set of sensors and is often performed during similar time periods of day.

This paper proposes an approach that enables smart home to become more context-aware. It recommends personalized actions to be performed for the user according to a current context (turning of lights when inhabitant is sleeping, turning off TV when inhabitant left the living room, turning on Radio when inhabitant enter the kitchen, etc...). The general purpose is to serve the user in a proactive and uninterrupted manner in various contexts that may arise.

\subsection{Architecture of the solution}

In the remainder of this section, we describe the functional architecture of our approach in order to show the role given to each of its modules "Fig. 2":

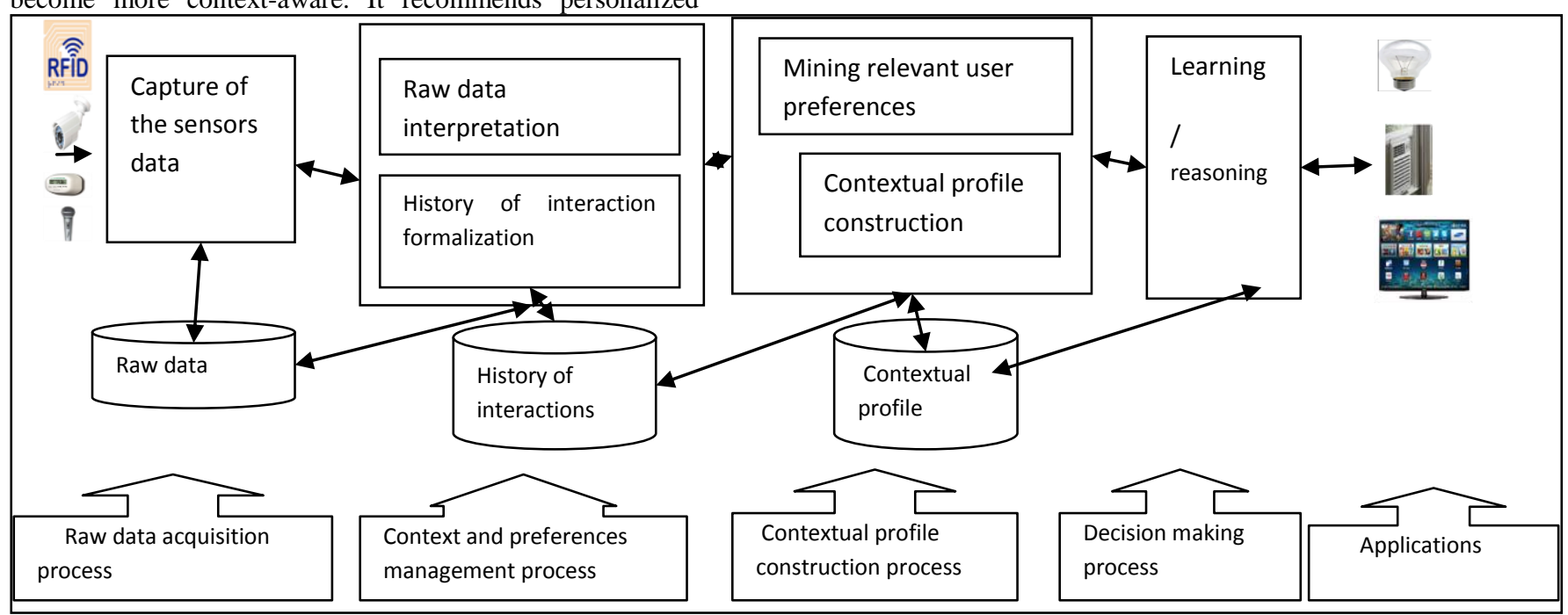

Fig 3: The general Architecture of the Proposed Solution

\subsubsection{Raw Data Acquisition Process}

This module is responsible of acquiring raw data from the physical context of the user and his interaction with PRS. It allows:

- The capture of the identity of the user from the RFID tags.

- The capture of physical context (temperature, light, etc.) using appropriate physical sensors for each type of information.

- The capture of user interactions: The interaction of the user with an PRS are explicit and/or implicit actions performed to satisfy a particular need. This captured information can be stored in log files, cookies, etc.

\subsubsection{Context and Preferences Management process}

The context management is the process of interpreting raw data (captured by physical sensors). These ones generally need to be transformed to be used directly by the application. In the context of our approach, this operation is done by the context and preferences management module. It allows to:

○ Identify the user;

- Identify the user context: the context identification consists of recognizing a contextual situation of the user.

- Extract user preferences: it consists of analyzing data that represent explicit and/or implicit interactions of the user with the PRS.
This analysis allows identifying user preferences.

- The History of interaction formalization: The user interaction with PRS will be stored in the historical interactions database using the tuple (user id, context, preference).

In terms of services, the user preferences are naturally affected by its context. These preferences differ from one context to another. For example, a user may prefer to listen to the radio in the morning and watching TV in the evening. So the preferences of a user are strongly related to his context and his profile.

We define the contextual profile of a user by his preference in a given context and we set it by the couple:

\section{Contextual profile $=($ Uid, user Context, user preference $)$}

In order to save the contextual user profile we must model these three components: user identifier, user context and user preference.

$$
\text { * UID }
$$

an attribute that identifies a user directly

\section{* User context}

We define the user context by all context parameters that may influence the user preferences in relation to an intelligent service. A context parameter $\left(\mathrm{P}_{\mathrm{i}}\right)$ is a parameter which represents fully or partially context information.

We use the model value-attribute to represent parameters of the context. The user context is represented as follows: 


\section{User Context $=\left\{P_{i} / i=1, \ldots ., n\right\}$}

$\mathrm{n}$ is the number of parameters that define a relevant context for an intelligent service.

In this study, we define the context of the user profile by:

User Context $=\{\mathrm{P} 0=$ Activity, $\mathrm{P} 1=$ Time, $\mathrm{P} 2=$ Localization, $\mathrm{P} 3=$ temperature, $\mathrm{P} 4=$ Special Event $\}$

Where attributes:

- P0: represents the human activity of the person (physical posture)

- $\quad$ P1 : represents the current time of the day $\{1-6,6-8$, $8-10,10-12,12-13,13-14,14-16,16-18,18-20,20$ $22,22-01\}$

- P2 : indicates the current location of the user \{kitchen, living room, bedroom, dining room \}

- P3 indicates the daytime temperatures \{cold, warm, hot $\}$

- And P4 indicate the name of the special event if it exists (weekend, Holiday, Sporting event, Cinema event, etc.).

\section{* User Preference}

We define a user preference with a set of descriptions including : what a user intends to do.

Example of some scenarios:

- The user is usually interested to political news. He prefers watching "France24" daily during the lunch break.

- In the afternoons during weekends, he watches sport programs.

Consequently, a user preference can be presented by the following parameters: The name of the service (TV, Radio, etc.), its associated type (Information, movies, documentaries, etc.) and the title of the chain or the radio station.

In general manner, we represent a user preference by:

\section{User preference $=\left\{\operatorname{Pref}_{j} / j=1, \ldots, \ldots, m\right\}$}

Where $\mathrm{m}$ is the number of parameters that define a set of descriptors, for this preference, in relation to an intelligent service.

In the considered smart home application, we define a service by 3 parameters: The service identifier, the state of the service (ON/OFF) and user preferences.
Where:

- $\quad$ Service-id : represents the name of a specific smart service: $\{$ Light, TV, air-conditioner ,etc... $\}$

- State: represents the state of the service : $\{\mathrm{ON}, \mathrm{OFF}\}$

- User Preferences $=\left\{\right.$ Pref $_{1}=$ Service name, Pref $_{2}=$ Associated type, Pref $_{3}=$ ChannelTitle $\}$

The user interaction with PRS will be stored in the historical interactions database using the couple (uid, context, preference).

\subsubsection{Contextual Profile Construction}

Only relevant preferences of the user will be considered to build his contextual profile. A preference is considered relevant if it is frequent, i.e its frequency exceeds a minimum threshold that we call MIN-FRQ-THRESHOLD.

To extract these relevant preferences, we applied the Apriori algorithm [19] on the user interaction preferences registered in the history of interactions database. Then, we combined these relevant preferences to their contexts (when they were occurring) and we save it in the user contextual profile database.

We note that this contextual profile database is periodically updated in order to track any eventual changes in user's behavior over time.

\subsubsection{Decision making process:}

In this layer, we use a three-layer neural network based on back propagation algorithm. This solution allows the system to consider the history of user experiences and calculate what will be the more suitable service to be applied in a given context.

Our network consists of three layers: The input that represents the data (context parameters), an output layer which represents the service (user preference), and a hidden layer which perform the intermediate calculations.

The purpose of the learning process is to optimize the network parameters to better predict user preferences in a given context. To do so, we try to reduce the error that represents the difference between the outputs produced by the network and the desired outputs. The produced errors will be corrected via the back-propagation method and weights of the network will be changed until convergence of the system.

The following table (table 2) shows some possible scenarios: 
Table 1: Some Scenarios of Trained Data

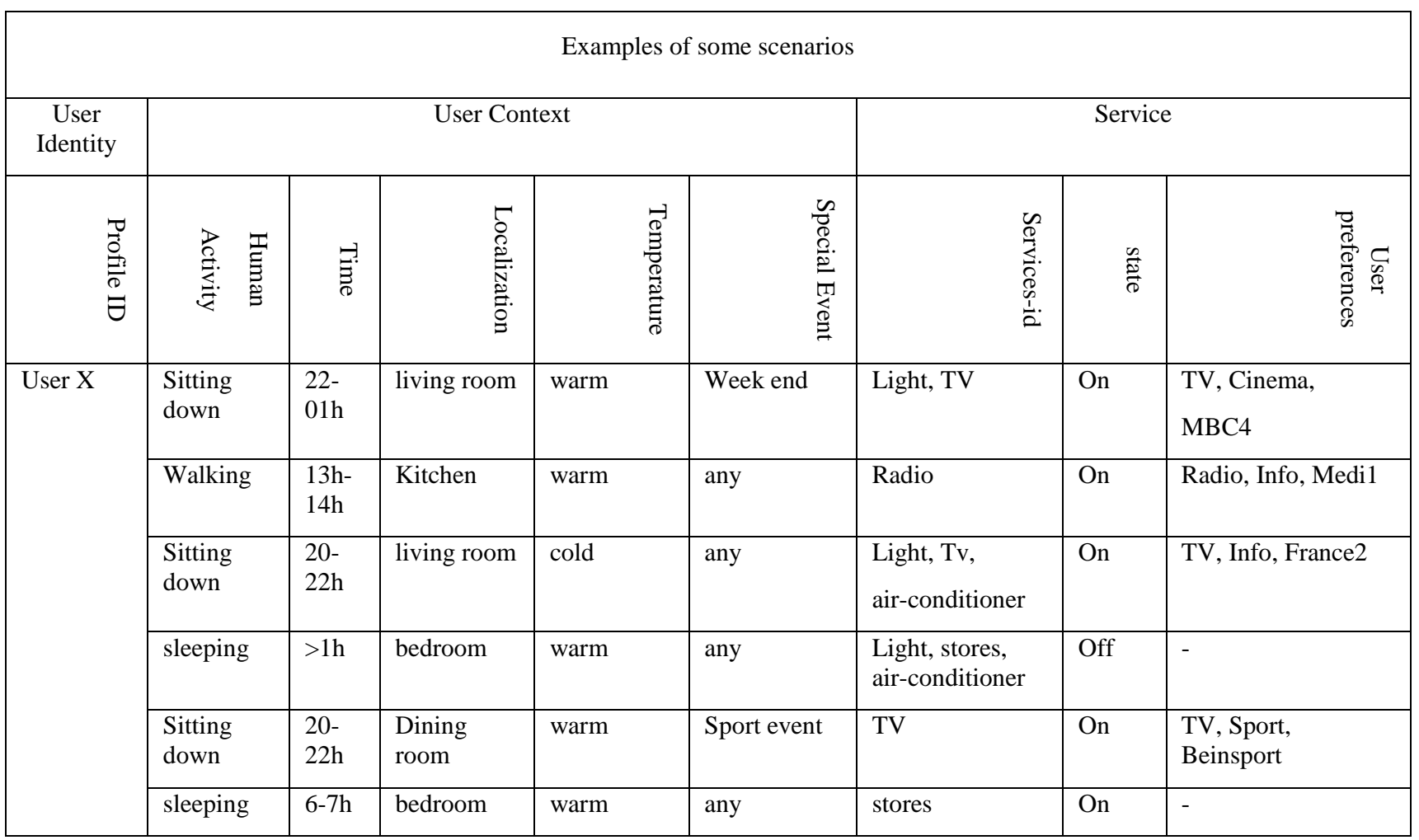

In our context, we consider the existence of only one person in the room at one time and we consider that the most significant activities (or human postures) are walking, sleeping, and sitting down.

The proposed system activates the appropriate candidate solutions (TV, radio, air-conditioner, stores, and lighting) according to a given context.

\section{CONCLUSION}

The main objective of building automation is to improve occupant comfort. The most existing solutions focus on recommending the most relevant items to users without taking into account the contextual information.

The general idea behind context aware systems is to facilitate user interaction with his external environment by determining, on behalf of the user, the application/service to be performed according to the user's situation, behaviors and preferences.

The aim of this paper was to incorporate the contextual information (such as current human activity, time, the presence of any special event,..) in the automatic recommendation process of smart home environment.

In the future studies we tend to address frequently encountered challenges in complex smart home environment like the presence of multiple users at the same time in a room and how to manage multiple activities occurring in a time period.

\section{ACKNOWLEDGMENTS}

Moroccan Government is actually working towards numerous major reforms to develop its own Smart City models. This work is part of the Casablanca smart city project aiming to support city systems and offer citizens a high quality of life.

\section{REFERENCES}

[1] Das S, Cook D, Battacharya A, Heierman E, Lin T. The role of prediction algorithms in the MavHome smart home architecture. IEEE Trans. On Wireless Communications, 2002,9(6):77-84.

[2] Gopalratnam K, Cook D J. Online sequential prediction via incremental parsing: The Active LeZi algorithm. IEEE Trans. On Intelligent Systems, 2007,22(1):52-58.

[3] Katharina R. Smart assistants for smart homes. Phd Thesis, 2013,Stockholm, Sweden.

[4] Gouttaya N, Belghini N , Begdouri A , Zarghili A. Improving the Proactive Recommendation in Smart Home Environments: An Approach Based on Case Based Reasoning and BP-Neural Network .I.J. Intelligent Systems and Applications, 2015, 07, 29-35.

[5] M. Montaner, B. López , and J. L. De La Rosa,"A Taxonomy of Recommender Agents on the Internet, Artificial Intelligence Review, pp. 285-330,June 2003.

[6] M. Weiser. The computer for the 21 st century. On Scientific American, 1991, 265(3): 94-104.

[7] R. Burke,"Hybrid Recommender Systems: Survey and Experiments",Journal of Personalization Research, User Modeling and User-Adapted Interaction,vol.12, pp. 331 - 370 , November 2002.

[8] T. Cioara, I. Anghel, I. Salomie, M. Dinsoreanu, G. Copil, and D. Moldovan, "A self-adapting algorithm for context aware systems," in Roedunet International Conference (RoEduNet), 2010 9th, june 2010, pp. 374 379 . 
[9] A K Dey, G D Abowd. "Towards a better understanding of context and context-awareness". Proceedings of the Workshop on the What, Who, Where, When and How of Context-Awareness. 2000, ACM Press, New York .

[10] J. Coutaz, J. Crowley, S. Dobson, and D. Garlan. "Context is key." Communications of the ACM 48(3), March 2005

[11] A. Manzoor, H.Truong, \& S.Dustdar. On the Evaluation of Quality of Context. In 3rd European Conference on Smart Sensing and Context pp. 140-153, 2008

[12] A.Held, S. Buchholz and A.Schill. Modeling of context information for pervasive computing applications. In Proceedings of SCI 2002/ISAS, 2002.

[13] Claudio Bettini, Oliver Brdiczka, Karen Henricksen, Jadwiga Indulska, Daniela Nicklas, Anand Ranganathan, Daniele Riboni, "A Survey of Context Modelling and Reasoning Techniques". Journal of Pervasive and Mobile Computing, 6(2):161-180, Elsevier, 2010.

[14] Ye et all., Situation identification techniques in pervasive computing: A review, Pervasive and Mobile Computing (2011), doi:10.1016/j.pmcj.2011.01.004

[15] T. van Kasteren, G. Englebienne, and B. Krose, "Activity recognition using semi-markov models on real world mart home datasets," J.Ambient Intell. Smart Environ. vol. 2, no. 3, 2010 .

[16] T. van Kasteren, G. Englebienne, and B. Kr"ose, "Transferring knowledge of activity recognition across sensor networks," in Pervasive, 2010.

[17] E. Hoque and J. Stankovic, "AALO: Activity recognition in smart homes using Active Learning in the presence of Overlapped activities," in Proc. of IEEE Int. Conf. on Pervasive Computing Technologies for Healthcare, San Diego, CA, 2012.

[18] M. A. Dragan and I. Mocanu, "Human Activity Recognition in Smart Environments," Control Systems and Computer Science (CSCS), 2013 19th International Conference on, Bucharest, 2013, pp. 495-502.

[19] M. A. Dragan and I. Mocanu, "Human Activity Recognition in Smart Environments," Control Systems and Computer Science (CSCS), 2013 19th International Conference on, Bucharest, 2013, pp. 495-502.

[20] R. Agrawal," Fast algorithms for mining association rules".Proceeding of the $20^{\text {th }}$ International Conference Very Large Data Bases, pp. 487-499, August 1994. 